\title{
Evaluación Clínica e Imagenológica de Dos Técnicas de Preservación de Reborde Alveolar Post Exodoncia
}

\author{
Clinical Assessment and Imaging of Two Preservation \\ Techniques of Alveolar Ridge Post Exodontia
}

Cristián López Riveros ${ }^{\star}$ \& Marcelo Ferrer Balart ${ }^{\star *}$

LÓPEZ, R. C. \& FERRER, B. M. Evaluación clínica e imagenológica de dos técnicas de preservación de reborde alveolar post exodoncia. Int. J. Odontostomat., 9(3):419-426, 2015.

RESUMEN: Ninguna técnica de preservación alveolar ha logrado conservar totalmente las dimensiones iniciales del reborde, luego de la extracción dentaria. Se ha reportado falta de evidencia científica y de estudios prospectivos que utilicen los alvéolos post exodoncia como control. El objetivo de este estudio fue comparar clínica e imagenológicamente las variaciones dimensionales del reborde óseo utilizando diferentes técnicas de preservación alveolar en un periodo de 6 a 8 semanas post exodoncia. Se recolectaron 10 pacientes con indicación de exodoncia de cuatro premolares por indicación de ortodoncia, usando un diseño de maxilares divididos. Un lado experimental (dos alvéolos) y el otro lado como control (dos alvéolos). Se observó que existieron diferencias significativas $(p<0,05)$ de reabsorción ósea entre el grupo experimental superior con el grupo control superior. El 33,3\% se los alvéolos experimentales presentaron algún tipo de exposición de membrana oclusiva, siendo la más frecuente la Matriz Dérmica Acelular (50\%). La técnica de exodoncia mínimamente traumática es la forma más simple y económica de preservar el reborde alveolar. No fue posible determinar cuál técnica es más eficiente para conservar las dimensiones del reborde alveolar.

PALABRAS CLAVE: técnica, preservación, alveolar, exodoncia.

\section{INTRODUCCIÓN}

El colapso de la pared bucal, ulterior a la pérdida dentaria, es un fenómeno que sigue en investigación, ya que los esfuerzos por mantenerla indemne, son poco predecibles. Los expertos sugieren la realización de estudios prospectivos, que usen el alveolo post exodoncia como control, e incentivan a seguir investigando sobre este asunto para proponer nuevos protocolos, y lograr conservar completamente las dimensiones del reborde alveolar luego de la extracción dentaria (Schropp et al., 2003; Cardaropoli \& Cardaropoli, 2008; Mezzomo et al., 2011; Morjaria et al., 2014).

Cuando un diente es extraído, y se ha planificado una rehabilitación en base a implantes, debemos considerar, que luego de la extracción dentaria, en casi la totalidad de los casos, se produce una pérdida en volumen del reborde alveolar, siendo más marcada la reabsorción en sentido buco-palatino que ápicocoronal (Lekovic et al., 1998), y mayor en el aspecto bucal que en el lingual/palatino, para el maxilar y la mandíbula (Schropp et al.; Araújo \& Lindhe, 2005).

La pérdida ósea en ancho del alveolo luego de la exodoncia, es de alrededor de un $50 \%$, de los cuales $2 / 3$ ocurren durante los 3 primeros meses, con una mayor reducción en la región de los molares comparado a la de los premolares, con mayor pérdida en la mandíbula que en el maxilar (Johnson, 1963, 1969; Pietrokovski \& Massler, 1967; Scropp et al.).

La técnica de exodoncia es preponderante en la futura reabsorción del reborde óseo, ya que esta acción constituye inevitablemente un trauma para los tejidos. Como también el instrumental seleccionado, el uso de colgajos y el manejo de la sutura (Araújo \& Lindhe, 2009; Oghli \& Steveling, 2010; Mezzomo et al.). "La forma más predecible de limitar la reabsorción ósea, y mantener las dimensiones del reborde alveolar,

* Cirujano Dentista, Magíster en Odontoestomatología, Universidad Andrés Bello, Santiago, Chile.

* Cirujano Dentista, Magíster en Periodoncia e Implantología. Docente de Pre grado y Post grado de Periodoncia e Implantología Universidad Andrés Bello, Santiago, Chile. 
es la preservación de este al momento de la exodoncia" (Keith \& Salama, 2007).

La preservación del reborde alveolar la podemos definir como: "Cualquier procedimiento que se lleva a cabo al momento de la extracción dentaria o consiguiente a ella, utilizado para limitar la reabsorción del reborde alveolar y maximizar la formación de tejido óseo dentro del alvéolo" (Darby et al., 2008). Por lo tanto, podemos considerar a la exodoncia mínimamente traumática como una técnica de preservación de reborde alveolar propiamente tal. Por otro lado, contamos con diversos biomateriales que podemos aplicar en el alvéolo para su conservación.

El objetivo de este estudio fue comparar clínica e imagenológicamente las variaciones dimensionales del reborde óseo utilizando diferentes técnicas de preservación alveolar en un periodo de 6 a 8 semanas post exodoncia.

\section{MATERIAL Y MÉTODO}

Selección de la muestra. Diez pacientes fueron seleccionados, hombres y mujeres, entre 13 y 20 años de edad (promedio de 13,9 años), con necesidad de exodoncia de 4 premolares por indicación de ortondoncia $(n=40)$. Fueron excluidos aquellos pacientes con enfermedad periodontal, reabsorción severa del hueso alveolar, ausencia de alguna pared del alveolo, enfermedad sistémica no controlada, enfermedad autoinmune, fumadores pesados (mayor a $10 \mathrm{ci}-$ garros/día), pacientes que hayan recibido radioterapia de cabeza y cuello en menos de 12 meses, consumo de medicamentos anticoagulantes, embarazadas, paciente con infarto al miocardio hace menos de 6 meses (Beitlitum et al., 2010). Este estudio fue aprobado por el comité de Bioética de la Facultad de Odontología de la Universidad Andrés Bello.

Todos los participantes firmaron previamente un consentimiento informado. Esta investigación consistió en realizar la exodoncia minimamente invasiva de todos los premolares, de los cuales la mitad fueron injertados con la combinación de dos tipos diferentes de hueso particulado y membranas de barrera, utilizando un mismo protocolo de injerto. Se utilizó un diseño de boca dividida. Nueve pacientes fueron derivados por el Instituto Nacional de Ortodoncia (INO) y un paciente por el post grado Ortodoncia de la Facultad de Odontología de la UNAB.
Todos los pacientes fueron sometidos a un examen clínico previo y a la toma de impresiones para modelos de estudio. Se efectuaron mediciones pre exodoncia; clínicas, en el modelo e imagenológicas, utilizando tomografía computadorizada Cone-beam.

1. Etapa pre-quirúrgica. Se establecieron 3 puntos de medición en la parte más central y prominente del reborde óseo, utilizando el margen gingival como referencia para la ubicación de estos puntos: i) Punto A, a $3 \mathrm{~mm}$ del margen gingival, ii) Punto $B$, a $6 \mathrm{~mm}$ del margen gingival y iii) Punto $\mathrm{C}$, a $9 \mathrm{~mm}$ del margen gingival.

Los puntos fueron trazados en el modelo, a nivel de cada premolar a extraer, y se trasladaron a un estampado rígido de acetato de cada paciente. Se perforó el estampado en los puntos determinados, el cual se utilizó para las mediciones clínicas e imagenológicas. La metodología de medición se aplicó en forma previa a la exodoncia, y 6 a 8 semanas después.

a) Mediciones en modelo. Se midió el espesor del reborde a nivel de los puntos mencionados con un caliper digital de hasta $0,01 \mathrm{~mm}$ de exactitud (Calibrador Micrométrico Digital de $150 \mathrm{~mm}$, RedLine Mechanics ${ }^{\circledR}$ ), y en las zonas retentivas del maxilar con un caliper análogo (EKSA) de hasta $0,25 \mathrm{~mm}$ de exactitud.

b) Mediciones imagenológicas. A cada paciente se le realizó un corte tomográfico previo, utilizando el estampado con conos de gutapercha como material radio opaco (Fig. 1A), en los puntos $A, B$ y $C$ de cada premolar por bucal y palatino/lingual (Fig. 1B). Para las mediciones en la tomografía se utilizó el software iCATVision $^{\text {Th }}$. Todas estas mediciones se realizaron a nivel de los 3 puntos $A$, B y $C$, en sentido horizontal y vertical (Fig. 2, Tabla I).

c) Mediciones Clínicas. Se utilizó Mepivacaína al 3\% como anestésico local, y se procedió a perforar la mucosa con una sonda para producir hemorragia en los puntos A, B y C, usando la plantilla como guía (Fig. 3).

2. Etapa pre-quirúrgica. Cada paciente recibió una profilaxis antibiótica con Amoxicilina de $1 \mathrm{~g}$, cada $12 \mathrm{~h}$ por 7 días, que comenzaron a tomar el día previo a la cirugía, junto con una profilaxis antinflamatoria de Ibuprofeno de 400 mg, cada 8 h por 3 días. Cada paciente realizó un enjuague con $10 \mathrm{ml}$ de gluconato de clorhexidina al $0,12 \%$ durante un minuto. 


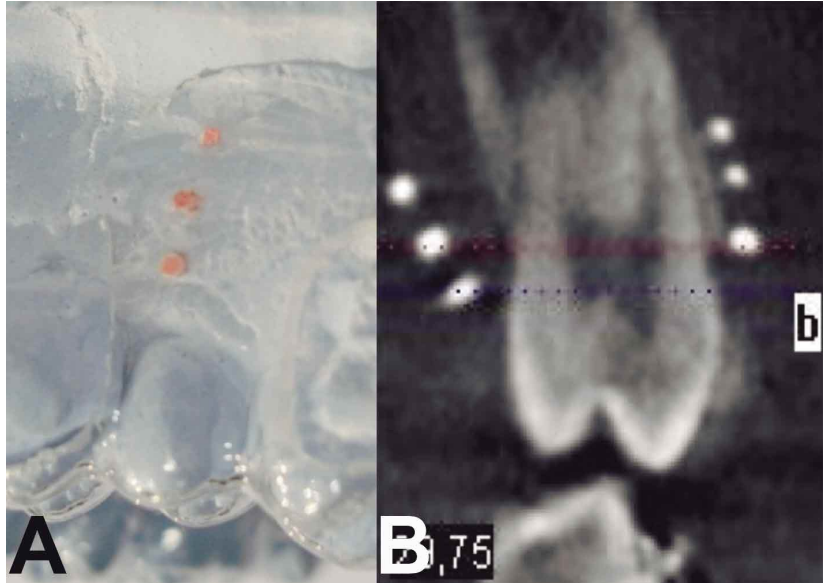

Fig. 1. A) Gutapercha como marcadores radio opacos. B) Marcadores radio opacos A, B y C bucales y palatinos.

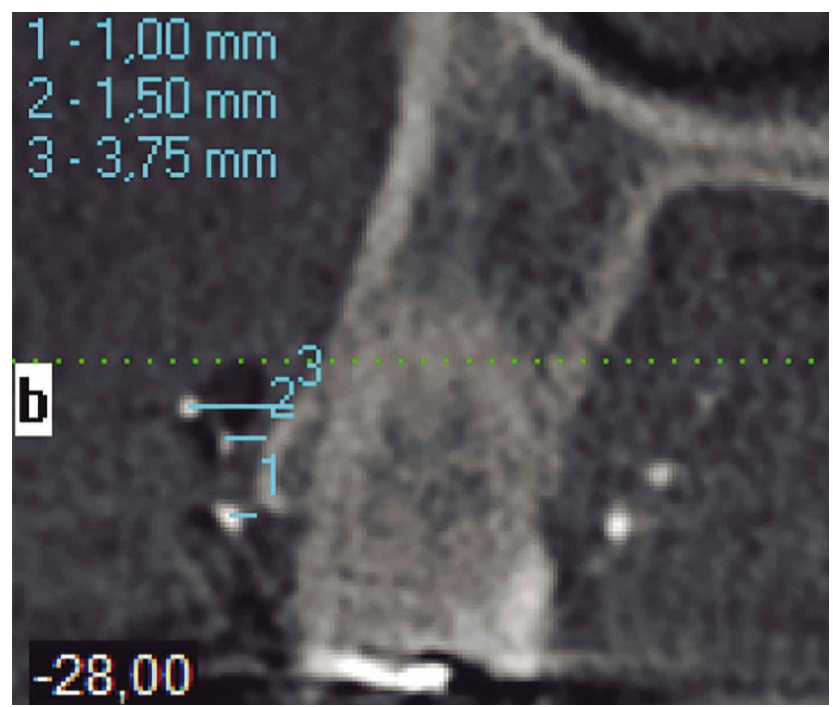

Fig. 2. Medición horizontal; marcadores bucales - tabla bucal (MB-TB).

Para este procedimiento se utilizó anestesia local, Cloruro de Mepivacaína al $2 \%$ con Corbadrina 1:20.000 (Mepi-Levo 20) y una box dental adaptado como sala de procedimientos.

a) Protocolo de exodoncia mínimamente invasiva. Ya anestesiado el paciente, se realizó la odontosección a nivel del LAC de los cuatro premolares. Luego con

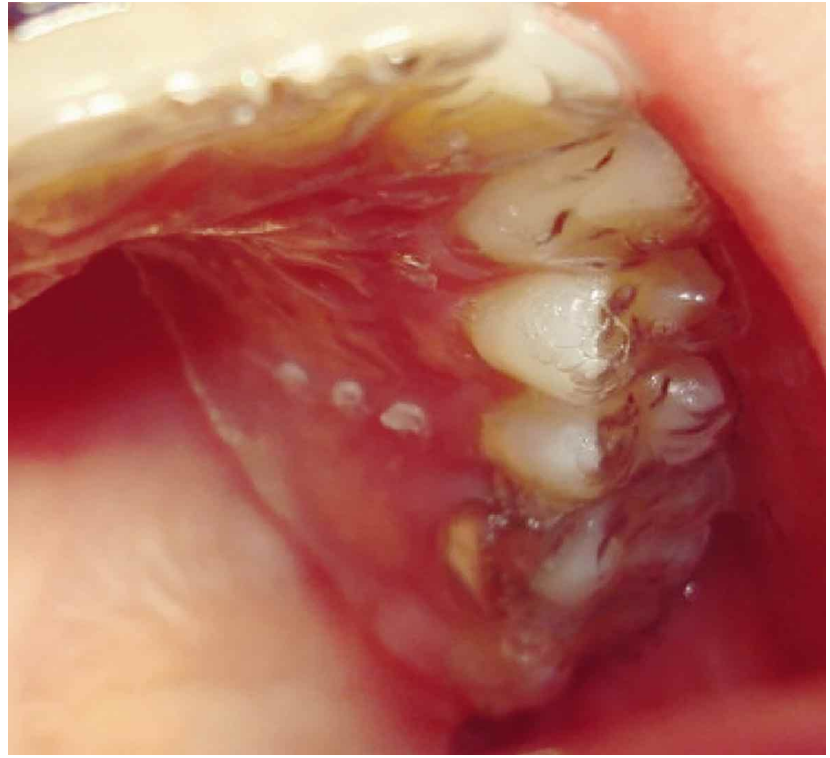

Fig. 3. Plantilla perforada puesta en boca guiando el sitio de punción.

el Kit de exodoncia mínimante traumático Neodent ${ }^{\circledR}$, se preparó el canal radicular con una broca helicoidal, para posteriormente anclar el traccionador del extractor dentario (Fig. 4A). Una vez anclado el traccionador dentro del canal radicular (Fig. 4B), se realizó la sindesmotomía de las fibras gingivales y periodontales con un Periotomo, alrededor de toda la raíz.

Se procedió a colocar el cable tractor estirado y perpendicular al eje mayor del extractor, y con un buen punto de apoyo sobre los dientes vecinos. Luego se gira la manilla leve y pausadamente en sentido horario, hasta que la raíz es extraída (Fig. 4C). Todas las exodoncias fueron realizadas por el mismo cirujano.

De los 40 dientes, sólo 9 se extrajeron utilizando el kit de exodoncia mínimamente traumático Neodent, debido a problemas técnicos que presentó el extractor dentario durante una de las exodoncias. El resto de los premolares fueron extraídos con técnica convencional, con movimientos principalmente de rotación y levemente buco-palatinos para premolares superiores, conservando ambas tablas óseas.

Tabla I. Mediciones realizadas en sentido horizontal y vertical en la Tomografía Computadorizada Cone-Beam.

\begin{tabular}{ll}
\hline Dimensión & Puntos de medición \\
\hline Horizontales & Marcador bucal $(\mathrm{MB})-$ Tabla bucal $(\mathrm{TB})$ \\
& Marcador palatino $(\mathrm{MP})-$ Tabla palatina/lingual $(\mathrm{TP})$ \\
& Tabla $(\mathrm{T})-$ Tabla $(\mathrm{T})$ \\
Verticales & Marcador bucal $(\mathrm{MB})-$ Cresta bucal $(\mathrm{CB})$ \\
& Marcador bucal $(\mathrm{MB})-$ Cresta palatina/lingual $(\mathrm{CP})$ \\
\hline
\end{tabular}




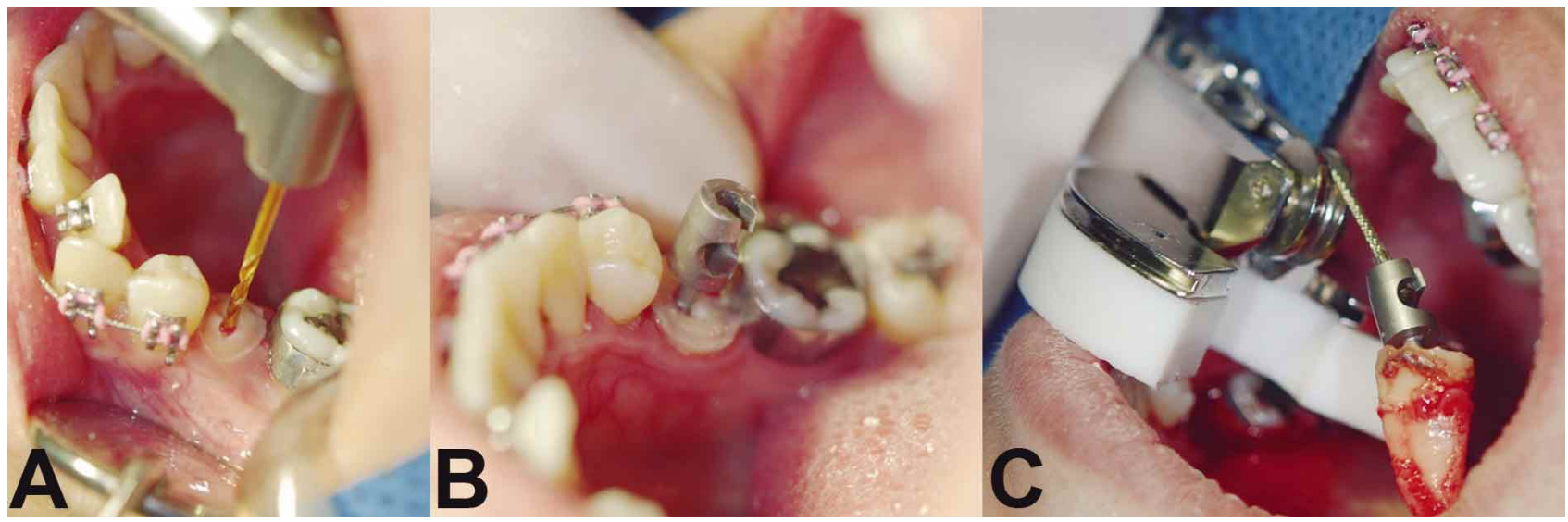

Fig. 4. A) Fresado del canal radicular con una broca helicoidal de 2,1 mm de diámetro, B) Traccionador anclado al interior del canal radicular, C) Avulsión de la raíz.

b) Protocolo de injerto. Una vez extraídos los cuatro premolares, se procedió a colocar el material de injerto en dos alvéolos homolaterales. Se utilizaron dos tipos de injertos óseos: FDBA (Freeze-Dried Bone Allograft) (MInerOss $®$ ) y Xenoinjerto bovino (Baumer®), y dos tipos de membranas de barrera: MDA (Matriz dérmica acelular) (Allodem GBR®) y Colágena (Baumer®).

Se limpió el alvéolo con gasa estéril, para remover el coágulo de sangre. Luego se abrió un colgajo bucal de espesor total, con una descarga mesial y otra distal, ambas entre las papilas adyacentes, abarcando solo el tejido blando del alveolo intervenido (Fig. $5 \mathrm{~A})$, con el objetivo de desplazar el colgajo coronalmente para lograr el cierre primario de la herida. Por palatino/lingual se abrió un bolsillo solamente legrando el mucoperiostio, sin realizar descargas. Después se procedió a empaquetar y condensar el injerto óseo particulado dentro del alvéolo, previamente hidratado con suero fisiológico (Fig. 5B). Una vez el tejido óseo particulado condensado en el alvéolo, se colocó la membrana correspondiente sobre el lecho injertado, cubriéndolo completamente, dejando uno de los extremos por dentro del bolsillo palatino/lingual, y el otro extremo por dentro del colgajo bucal (Fig. 5C) con desplazamiento coronal, quedando ambos extremos de la membrana entre la pared ósea externa del alveolo y la cara interna del mucoperiostio.

En todos los alvéolos injertados se realizó el mismo protocolo de injerto, utilizando un colgajo desplazado para lograr un cierre primario y dejar las membranas sumergidas. Una vez suturados los alvéolos injertados, se procedió a dejar un cemento quirúrgico (Coe Pak ${ }^{\circledR}$ ) sobre los alvéolos experimentales, por un periodo de una semana. Los alvéolos control, se dejaron cicatrizar en forma normal.

\section{Controles post exodoncia.}

$1^{\text {a }}$ semana: Se controló mediante anamnesis y exa-

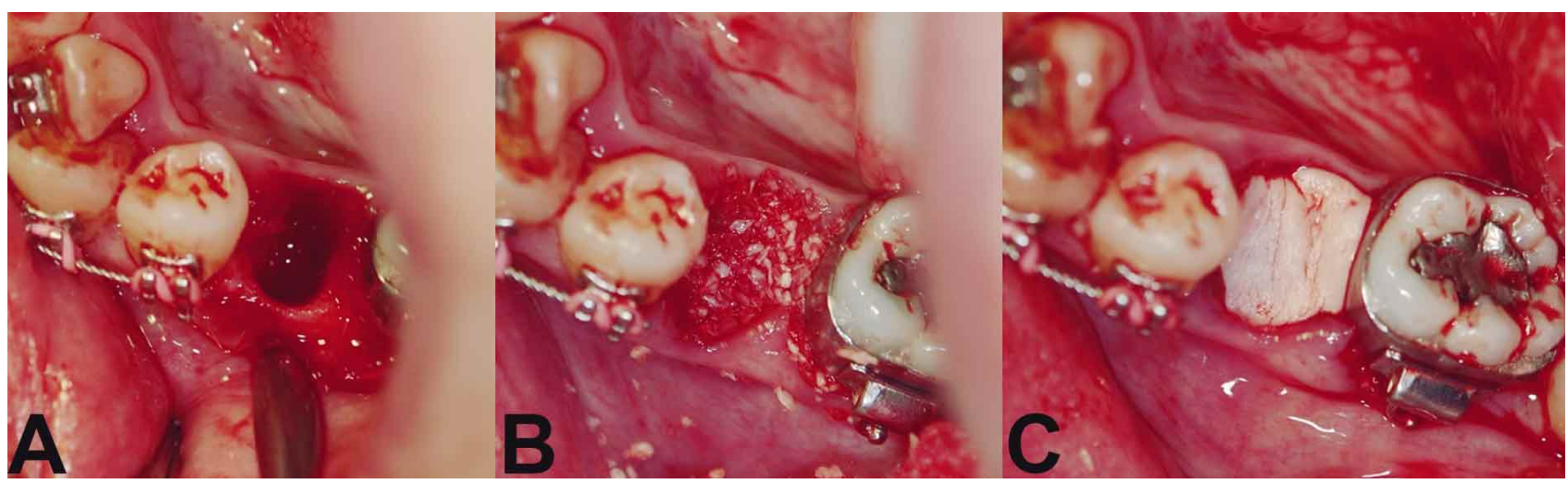

Fig. 5. A) Colgajo bucal de espesor total. Se observa la integridad de la cortical bucal, B) Injerto óseo particulado condensado al interior del alveolo, C) Membrana de barrera cubriendo completamente el alvéolo experimental. 
men clínico la cicatrización, signos y síntomas inflamatorios, complicaciones post exodoncia, incorporación de la membrana e injerto por los tejidos, entre otras, además de retirar el cemento quirúrgico.

$2^{\mathrm{a}}$ semana: Se controlaron los mismos parámetros que en la primera semana, además de retirar las suturas (Fig. 6).

$6^{\mathrm{a}}$ a $8^{\mathrm{a}}$ semanas: Se realizaron los controles finales post exodoncia, con un nuevo corte tomográfico, mediciones clínicas e impresiones para modelos de estudio, siguiendo el mismo protocolo descrito anteriormente para cada etapa. Se solicitó a los ortodoncistas tratantes la mantención de los arcos seccionales para poder realizar el respectivo control (Fig. 6).

De los 10 pacientes que formaron parte de este estudio, solo 6 pudieron ser controlados en este periodo. Los cuatro pacientes restantes fueron excluidos de este estudio, debido a falta de tiempo para su control final post exodoncia con mediciones clínicas, imagenológicas y modelos de estudio. No alcanzaron a ser controlados dentro de la fecha estimada para la ejecución de esta investigación. Para términos de evaluar y comparar las complicaciones post exodoncia e injerto, se incluyeron los 10 pacientes de la muestra inicial, ya que todos pudieron ser controlados en un periodo de dos semanas para ver dichos resultados

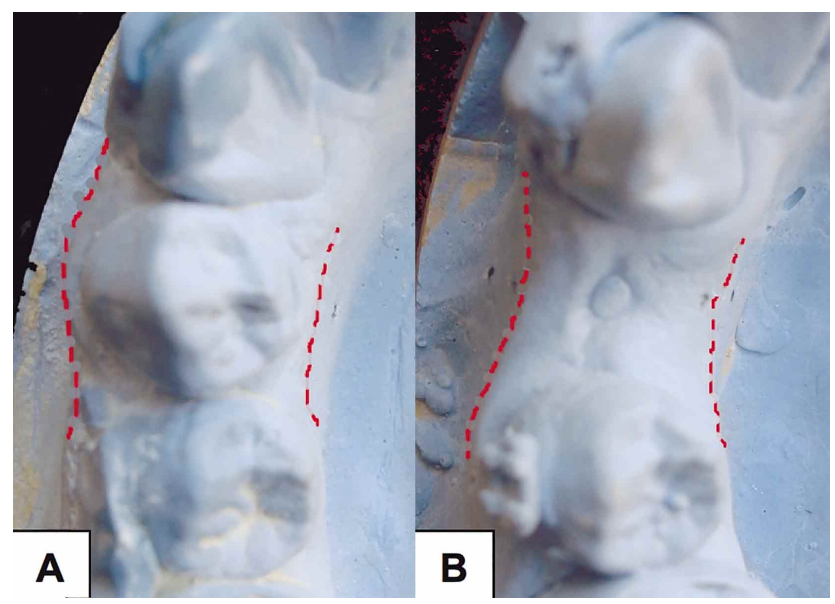

Fig. 6. Contorno (en rojo) del reborde alveolar en sitio control inferior. Comparación de antes (A) y 6 semanas después de la exodoncia (B). Se aprecia el adelagazamiento del contorno en $\mathrm{B}$.

\section{RESULTADOS}

Los datos primarios fueron comparados entre el grupo control y experimental en todas las variables den- tro de cada método estudiado. Dichas comparaciones se realizaron mediante la prueba de rangos de Wilcoxon para dos muestras relacionadas. El nivel de significación empleado fue de $\partial \leq 0,05$ en todos los casos.

Se observaron diferencias estadísticamente significativas $(p<0,05)$ en el método imagenológico entre las medianas de reabsorción ósea horizontal del grupo experimental y control superior en: MB-TB en el punto $A, T-T$ en los puntos $A$ y $B$, y en MP-TP en el punto $\mathrm{C}$. Se observó un mayor nivel de reabsorción en el grupo experimental superior a nivel de los puntos mencionados anteriormente comparado a los controles contralaterales.

No se observaron resultados estadísticamente significativos en resto de las variables en los otros métodos utilizados.

De las complicaciones post quirúrgicas, se observó que la exposición de membrana fue la mas común. Donde la más frecuente fue en el grupo injertado con matriz dérmica acelular $(50 \%$ de los casos injertados con MDA presentó algún tipo de exposición, y un $80 \%$ sintió mal olor y mal sabor asociado).

\section{DISCUSIÓN}

La exodoncia mínimamente invasiva es el método más simple que se puede emplear para conservar las dimensiones del reborde alveolar. El uso de injertos óseos podría resultar en una menor disminución del contorno del reborde alveolar luego de la exodoncia, especialmente de la pared bucal (Fickl et al., 2008). Sin embargo, en el presente estudio se pudo observar que el grupo experimental presentó una mayor reabsorción a nivel marginal de la tabla ósea bucal (punto A) que el grupo control, al análisis imagenológico. Lo cual se condice con la diferencia significativa de reabsorción del ancho del alveolo a nivel del tercio marginal y medio (puntos $A$ y $B$ ) del grupo experimental comparado con el grupo control, del maxilar.

La mayor reabsorción de la pared ósea bucal (tercio marginal y medio) tiene relación con lo señalado en estudios anteriores de Pietrokovski \& Massler, Cardaropoli et al. (2003), Schropp et al., Araújo \& Lindhe (2005), Barone et al. (2008) y Tan et al. (2011). Donde corroboran que existe una mayor reabsorción ósea en el aspecto bucal que en el aspecto palatino. 
Sin embargo, al comparar los niveles de reabsorción entre ambos grupos, se obtuvo resultados no esperados que discrepan de la literatura, ya que se reporta una mayor pérdida ósea a nivel de los alvéolos superiores que fueron injertados comparado a los controles superiores. Donde podríamos cuestionar si efectivamente es útil aplicar un injerto adicional a la técnica de exodoncia minimamente invasiva. Algo muy diferente observó Lekovic et al. (1998) donde utilizó membranas reabsorbibles de polímeros glicólicos y lácticos en alvéolos experimentales, que resultaron tener una menor pérdida ósea en ancho respecto de los controles.

En el presente estudio, esta mayor pérdida ósea en el grupo experimental se podría atribuir a: 1) El uso de un colgajo de espesor total desplazado coronalmente (Araújo \& Lindhe, 2009), 2) Gran parte de los pacientes presentaban un biotipo gingival fino, 3) La tensión generada por la sutura para lograr el cierre primario (McAllister \& Haghighat 2007; Oghli \& Steveling; Mezzomo et al.).

Sin embargo, en la mandíbula no se observó diferencias significativas de reabsorción entre ambos grupos, pese a que en el análisis visual de los rebordes se apreció un mayor colapso de la tabla bucal en el lado control. Esto es producto de que la membrana de barrera utilizada además de evitar la invasión del tejido conectivo y del epitelio al interior del alveolo, genera un "engrosamiento" del tejido gingival, dando un aspecto de un reborde más ancho, que tiende a enmascarar clínicamente el espesor real del reborde óseo, lo cual fue más notorio en los casos en que se utilizó MDA, siendo también una de las membranas con mayor tasa de exposición en esta investigación (50\%). Ocho de diez pacientes relataron haber sentido mal olor y mal sabor en presencia de este tipo de membrana, lo que podría estar relacionado con su exposición e infección, ya que al retirarla (en los casos de exposición total), cesó el mal olor y el mal sabor.

Gran parte de los estudios referentes a este tema realizan controles post exodoncia/técnica de preservación después de los tres meses. En este periodo se producen los mayores cambios del reborde óseo (Camargo et al., 2000; lasella et al., 2003; Schropp et al.; Chen et al., 2004; Drakos \& Engler-Hamm, 2006). Pese a que esta investigación está hecha con un diseño de control post exodoncia de seis a ocho semanas, ya se puede observar diferencias significativas en las magnitudes de reabsorción horizontal entre los alvéolos superiores control y experimental. Lo cual puede ser atribuido a que el material de injerto/membrana de barrera podrían estar influyendo en el proceso de cicatrización y reabsorción ósea en forma negativa (Hämmerle \& Jung, 2003), o simplemente es necesario esperar más tiempo para observar mayores cambios en el grupo control, teniendo en cuenta que la reabsorción ósea es un proceso que se prolonga hasta después de los 12 meses post exodoncia (Johnson, 1969; Lekovic et al., 1997; Schropp et al.; Morjaria et al.).

Se pudo apreciar mayor reabsorción ósea a nivel del tercio apical de la tabla palatina de los alvéolos experimentales superiores comparado con los controles superiores, un resultado inesperado, ya que es la tabla con menor susceptibilidad de reabsorción, por el grosor que presenta en el tercio mas apical, y es la zona en donde el coágulo se diferencia más rápido en tejido óseo, por la proximidad de las corticales del alveolo, permitiendo que este proceso sea más expedito (Cardaropoli et al.; Araujo \& Lindhe, 2009), haciendo que el comportamiento de esta cortical sea más predecible que el de la cortical bucal frente a la exodoncia.

Con las observaciones realizadas se puede establecer que la hipótesis planteada para este estudio es rechazada, ya que las diferencias estadísticamente significativas, se presentaron sólo en el método imagenológico, y dictan a favor de la técnica de exodoncia minimamente traumática (grupo control). No se encontraron diferencias estadísticamente significativas para los otros dos métodos empleados, por lo que no se puede determinar cuál técnica preserva el reborde alveolar en mayor cuantía. Por ende, se cree necesario realizar un seguimiento de más tiempo para evaluar si los resultados se revierten o siguen el mismo patrón obtenido hasta ahora.

Las principales limitaciones de este estudio fueron el número de la muestra para cada técnica, el tiempo de evaluación post exodoncia, y que se utilizaron muchos tipos de injertos.

Otra limitación que se presentó, muy similar a lo que ocurrió en el estudio de Fickl et al., es que; se utilizaron modelos para analizar la pérdida ósea horizontal, por lo tanto, no es posible asegurar que las variaciones dimensionales del ancho del reborde sean producto de cambios en el tejido blando o por reabsorción del tejido óseo subyacente. 
Por otro lado, el método clínico utilizado tampoco fue el más fidedigno, ya que el calibrador no despreciaba totalmente el grosor de la mucosa en las mediciones clínicas, pudiendo dar medidas de espesor levemente mayores a las reales.

\section{CONCLUSIONES}

La técnica más simple y económica de preservar el reborde alveolar es la exodoncia mínimamente traumática, que si bien, no permite conservar en su totalidad las dimensiones del reborde, logra limitar una reabsorción ósea más severa.
Los resultados indican que la exodoncia minimamente traumática sola entrega un mejor costo/ beneficio, ya que ningún alvéolo control presentó complicaciones post quirúrgicas, no así los alvéolos experimentales que presentaron en varios casos exposición de la membrana oclusiva (33,3\%), siendo la Matriz Dérmica Acelular la con mayor tasa de exposición (50\%).

Con los resultados obtenidos en esta investigación no es posible determinar cuál técnica es más eficiente en la preservación de la tabla bucal debido a que la muestra es muy pequeña y el tiempo de evaluación post exodoncia sigue siendo muy limitado para observar cambios acentuados en el reborde alveolar.

LÓPEZ, R. C. \& FERRER, B. M. Clinical assessment and imaging of two preservation techniques of alveolar ridge post exodontia. Int. J. Odontostomat., 9(3):419-426, 2015.

ABSTRACT: No ridge preservation technique has achieved to fully sustain the initial dimensions of the alveolar bone at the moment of tooth extraction. Lack of scientific evidence and prospective studies that use the alveolus as control site have been reported. The aim of this study was to clinically and imagenologically compare the variation of the alveolar ridge dimensions, using different alveolar ridge preservation techniques in a period of 6 to 8 weeks post tooth extraction. Ten (10) patients with orthodontic tooth extraction indication of four premolars were collected, using a split mouth design. An experimental side (two sockets) and the other side as control (two sockets). There were significant differences of bone resorption between the superior experimental group and the superior control group. 33, 3\% of the experimental alveolus showed some kind of barrier membrane exposure, being Acellular Dermal Matrix (ADM) the most frequent (50\%). The atraumatic extraction technique is the most simple and economic way of preserving the alveolar ridge. It was not possible to determine which technique is the most efficient to conserve la alveolar ridge dimensions.

KEY WORDS: technique, preservation; alveolar, tooth extraction.

\section{REFERENCIAS BIBLIOGRÁFICAS}

Araújo, M. G. \& Lindhe, J. Dimensional ridge alterations following tooth extraction. An experimental study in the dog. J. Clin. Periodontol., 32(2):212-8, 2005.

Araújo, M. G. \& Lindhe, J. Ridge alterations following tooth extraction with and without flap elevation: an experimental study in the dog. Clin. Oral Implants Res., 20(6):5459, 2009.

Barone, A.; Aldini, N. N.; Fini, M.; Giardino, R.; Calvo Guirado, J. L. \& Covani, U. Xenograft versus extraction alone for ridge preservation after tooth removal: a clinical and histomorphometric study. J. Periodontol., 79(8):1370-7, 2008.

Beitlitum, I.; Artzi, Z. \& Nemcovsky, C. E. Clinical evaluation of particulate allogeneic with and without autogenous bone grafts and resorbable collagen membranes for bone augmentation of atrophic alveolar ridges. Clin. Oral Implants Res., 21(11):1242-50, 2010.
Camargo, P. M.; Lekovic, V.; Weinlaender, M.; Klokkevold, P. R.; Kenney, E. B.; Dimitrijevic, B.; Nedic, M.; Jancovic, S. \& Orsini, M. Influence of bioactive glass on changes in alveolar process dimensions after exodontia. Oral Surg. Oral Med. Oral Pathol. Oral Radiol. Endod., 90(5):581-6, 2000.

Cardaropoli, G.; Araújo, M. \& Lindhe, J. Dynamics of bone tissue formation in tooth extraction sites. An experimental study in dogs. J. Clin. Periodontol., 30(9):809-18, 2003.

Cardaropoli, D. \& Cardaropoli, G. Preservation of the postextraction alveolar ridge: a clinical and histologic study. Int. J. Periodontics Restorative Dent., 28(5):46977, 2008.

Chen, S. T.; Wilson, T. G. Jr. \& Hämmerle, C. H. Immediate or early placement of implants following tooth extraction: review of biologic basis, clinical procedures, and outcomes. Int. J. Oral Maxillofac. Implants, 19 (Suppl.):12-25, 2004. 
Darby, I.; Chen, S. \& De Poi, R. Ridge preservation: what is it and when should it be considered. Aust. Dent. J., 53(1):11-21, 2008.

Drakos, D. \& Engler-Hamm, D. Ridge preservation: why and when. Implants, 3(1):20-3, 2006.

Fickl, S.; Zuhr, O.; Wachtel, H.; Stappert, C. F.; Stein, J. M. \& Hürzeler, M. B. Dimensional changes of the alveolar ridge contour after different socket preservation techniques. J. Clin. Periodontol., 35(10): 906-13, 2008.

Hämmerle, C. H. \& Jung, R. E. Bone augmentation by means of barrier membranes. Periodontol. 2000, 33:36-53, 2003.

preservation with freeze-dried bone allograft and a collagen membrane compared to extraction alone for implant site development: a clinical and histologic study in humans. J. Periodontol., 74(7):990-9, 2003.

Johnson, K. A study of the dimensional changes occurring in the maxilla following tooth extraction-Part 1. Normal healing. Aust. Dent. J., 8: 428-433, 1963.

Johnson, K. A study of the dimensional changes occurring in the maxilla following tooth extraction. Aust. Dent. J., 14(4):241-4, 1969.

Keith, J. D. Jr. \& Salama, M. A. Ridge preservation and augmentation using regenerative materials to enhance implant predictability and esthetics. Compend. Contin. Educ. Dent., 28(11):614-21, 2007.

Lekovic, V.; Kenney, E. B.; Weinlaender, M.; Han, T.; Klokkevold, P.; Nedic, M. \& Orsini, M. A bone regenerative approach to alveolar ridge maintenance following tooth extraction. Report of 10 cases. J. Periodontol., 68(6):56370,1997 .

Lekovic, V.; Camargo, P. M.; Klokkevold, P. R.; Weinlaender, M.; Kenney, E. B.; Dimitrijevic, B. \& Nedic, M. Preservation of alveolar bone in extraction sockets using bioabsorbable membranes. J. Periodontol., 69(9):10449, 1998.

McAllister, B. S. \& Haghighat, K. Bone augmentation techniques. J. Periodontol., 78(3):377-96, 2007.

Mezzomo, L. A.; Shinkai, R. S.; Mardas, N. \& Donos, N. Alveolar ridge preservation after dental extraction and before implant placement: a literature review. Rev. Odonto. Cienc., 26(1):77-83, 2011.

Morjaria, K. R.; Wilson, R. \& Palmer, R. M. Bone healing after tooth extraction with or without an intervention: a systematic review of randomized controlled trials. Clin. Implant. Dent. Relat. Res., 16(1):1-20, 2014.
Oghli, A. A. \& Steveling, H. Ridge preservation following tooth extraction: a comparison between atraumatic extraction and socket seal surgery. Quintessence Int., 41(7):6059, 2010.

Pietrokovski, J. \& Massler, M. Alveolar ridge resorption following tooth extraction. J. Prosthet. Dent., 17(1):217,1967 .

Schropp, L.; Wenzel, A.; Kostopoulos, L. \& Karring, T. Bone healing and soft tissue contour changes following single-tooth extraction: a clinical and radiographic 12-month prospective study. Int. J. Periodontics Restorative Dent., 23(4):313-23, 2003.

Tan, W. L.; Wong, T. L.; Wong, M. C. \& Lang, N. P. A systematic review of post-extractional alveolar hard and soft tissue dimensional changes in humans. Clin. Oral Implants Res., 23 (Suppl. 5):1-21, 2012.

Dirección para Correspondencia:

Cristián López Riveros

Cirujano Dentista

Magíster en Odontoestomatología

Universidad Andrés Bello

Santiago

CHILE

Email: cilopezriveros@gmail.com

Recibido: 07-06-2015

Aceptado: 06-10-2015 